Open Access

Original Article

\title{
High prevalence of multidrug resistant uropathogens: A recent audit of antimicrobial susceptibility testing from a tertiary care hospital in Bangladesh
}

\author{
Chandrika Dasgupta ${ }^{1}$, Md. Abdur Rafi' ${ }^{2}$ Md. Abdus Salam ${ }^{3}$
}

\begin{abstract}
Objectives: Urinary tract infections due to multi drug resistant bacteria have been on the rise globally with serious implications for public health. The objective of this study was to explore the prevalence of multi drug resistant uropathogens and to correlate the urinary tract infections with some demographic and clinical characteristics of patients admitted in a tertiary care hospital in Bangladesh.

Methods: A cross sectional prospective study was conducted at Shaheed Ziaur Rahman Medical College Hospital, Bogura, Bangladesh among clinically suspected urinary tract infection patients from January to December, 2018. Clean-catch midstream or catheter-catch urine samples were subjected to bacteriological culture using chromogenic agar media. Antimicrobial susceptibility testing of the isolates was done by KirbyBauer disk diffusion method following Clinical and Laboratory Standards Institute guidelines. Descriptive statistical methods were used for data analysis.

Results: Culture yielded a total of 537 (42.8\%) significant bacterial growths including $420(78.2 \%)$ multi drug resistant uropathogens from 1255 urine samples. Escherichia coli was the most common isolate (61.6\%) followed by Klebsiella spp. (22.5\%), Pseudomonas spp. (7.8\%), Staphylococcus aureus (5.4\%) and Enterobacter spp. (2.6\%) with multi drug resistance frequency of $77.6 \%, 71.9 \%, 90.5 \%, 86.2 \%$ and $92.9 \%$ respectively. There was female preponderance (M:F; 1:1.97; $\mathrm{P}=0.007)$ but insignificant differences between paediatric and adult population (43.65\% vs. $42.57 \%$ ) and also among different age groups. Diabetes, chronic renal failure, fever and supra-pubic pain had significant association as co-morbidities and presentations of urinary tract infections $(\mathrm{P}<0.05)$. Multi drug resistance ranged from 3.7 to $88.1 \%$ including moderate to high resistance found against commonly used antibiotics like ciprofloxacin, cephalosporin, azithromycin, aztreonam, cotrimoxazole and nalidixic acid (28.6 to $92.9 \%$ ). Isolates showed 2.4 to $32.2 \%$ resistance to nitrofurantoin, amikacin, netilmicin and carbapenems except Pseudomonas spp. (66.7\% resistance to nitrofurantoin) and Enterobacter spp. (28.6 to $42.9 \%$ resistance to carbapenems).

Conclusion: There is very high prevalence of multi drug resistant uropathogens among hospitalized patients and emergence of carbapenem resistance is an alarming situation. Antibiotic stewardship program is highly recommended for hospitals to combat antimicrobial resistance.
\end{abstract}

KEYWORDS: Antibiogram, Multidrug resistant uropathogens, Tertiary care hospital, Bangladesh.

doi: https://doi.org/10.12669/pjms.36.6.2943

How to cite this:

Dasgupta C, Rafi MA, Salam MA. High prevalence of multidrug resistant uropathogens: A recent audit of antimicrobial susceptibility testing from a tertiary care hospital in Bangladesh. Pak J Med Sci. 2020;36(6):1297-1302. doi: https://doi.org/10.12669/pjms.36.6.2943

This is an Open Access article distributed under the terms of the Creative Commons Attribution License (http://creativecommons.org/licenses/by/3.0), which permits unrestricted use, distribution, and reproduction in any medium, provided the original work is properly cited.

Correspondence:

Dr. Md. Abdus Salam

E-mail: abdussalam@iium.edu.my

* Received for Publication

* $1^{\text {st }}$ Revision Received:

* $2^{\text {nd }}$ Revision Received:

* Final Revision Accepted:
May 21, 2020

June 15, 2020

July 20, 2020

July 25, 2020

\section{INTRODUCTION}

Urinary tract infection (UTI) is among the most frequent bacterial infections in clinical practice worldwide. The frequency and burden of UTI is mostly underestimated and speculated to be higher than available data because it is not among 
mandatory notifiable diseases. ${ }^{1}$ A spectrum of clinical scenario from asymptomatic bacteriuria to complicated infections are observed in UTI affecting different age groups. Dysuria with or without frequency, urgency, and suprapubic pain are the usual accompaniments of lower urinary tract infections while complicated UTI like pyelonephritis usually presents with systemic symptoms like fever, chill, flank pain, hematuria and delirium. ${ }^{2}$ Escherichia coli remains the predominant uropathogen $(80 \%)$ followed by Klebsiella, Enterobacter, Proteus, Pseudomonas and Enterococci. The pathogens traditionally associated with UTI are on a change particularly because of growing antimicrobial resistance and underlying host factors. ${ }^{3}$

UTI is becoming increasingly difficult to treat owing to high recurrence and multi drug resistant (MDR) uropathogens especially extendedspectrum $\beta$-lactamases (ESBLs) producing bacteria that have been on the rise globally with serious implications for public health. ${ }^{4}$ Traditionally broad spectrum antibiotics remain the drug of choice to treat UTI but indiscriminate use of antibiotics is making the treatment challenging as it accelerates the emergence of drug resistant bacteria. This practice is more common in lower and middle income countries like Bangladesh, where empirical therapy is a common practice as the laboratory facility for urine culture is not widely available. ${ }^{5}$ Although bacterial etiology of UTI and antimicrobial resistance pattern may have regional variations and largely depend on the antibiotic policy of health care facility but the growing frequency of MDR uropathogens has become now a universal problem and more alarming in the developing countries including Bangladesh. ${ }^{6,7}$

Routine antimicrobial susceptibility testing is a pre-requisite not only to choose the appropriate antibiotic but also to facilitate the empiric therapy. ${ }^{8}$ The present investigation was carried out to explore the current prevalence of MDR uropathogens, frequency of resistance to different classes of antibiotics and to correlate UTI with patient's demographic and clinical characteristics from a tertiary care hospital in Bangladesh.

\section{METHODS}

This cross sectional prospective investigation was conducted from January to December, 2018 among one thousand two hundred fifty-five (1255) admitted patients of different age and gender of
Shaheed Ziaur Rahman Medical College Hospital (SZMCH), Bogura, a 1000-bed tertiary care teaching hospital in the Northern part of Bangladesh. Patients with at least one of the clinical features of UTI (fever, dysuria, frequency, urgency, suprapubic pain, loin pain, haematuria, nocturia or prior history of UTI) were judged as clinically suspected and selected for confirmation by laboratory tests. Clean-catch midstream or catheter-catch urine was collected into a sterile wide mouth container/test tube with all aseptic measures and was screened for microscopic demonstration of pus cells $\geq 5$ / HPF (high power field) in a centrifuged deposit before considering culture. ${ }^{9}$

Ethical Review Committee of Shaheed Ziaur Rahman Medical College, Bogura, Bangladesh approved the protocol and informed written consent/assent was taken from patient (Ref: SMZC/2016/351, Dated: 10-12-2016).

Urine culture: Chromogenic agar (HiMedia, India) medium was used for urine culture and samples were inoculated aseptically using a calibrated wire loop of 28G (internal diameter $3.26 \mathrm{~mm}$ ) holding $0.004 \mathrm{ml}$ of urine for overnight aerobic incubation at $37^{\circ} \mathrm{C}$. Details of culture technique, significant bacteriuria and identification methods have been reported previously. ${ }^{10}$ The final identification of the isolates was done using standard identification protocol such as Gram's staining, motility test, catalase test, coagulase test, oxidase test and relevant biochemical tests as appropriate for the isolates. ${ }^{11}$ Diagnosis of UTI was established on the basis of presenting feature(s), pyuria and significant bacteriuria.

Antimicrobial susceptibility testing (AST): Mueller-Hinton agar and Kirby-Bauer disk diffusion method ${ }^{12}$ were used for AST against a panel of 17 commercial antibiotic disks (Oxoid, $\mathrm{UK})$ : amikacin $(30 \mu \mathrm{g})$, azithromycin $(15 \mu \mathrm{g})$, aztreonam $(30 \mu \mathrm{g})$, cefuroxime sodium $(30 \mu \mathrm{g})$, cefixime $(5 \mu \mathrm{g})$, cefepime $(30 \mu \mathrm{g})$, ceftazidime $(30 \mu \mathrm{g})$, ceftriaxone $(30 \mu \mathrm{g})$, ciprofloxacin $(10 \mu \mathrm{g})$, cotrimoxazole $(25 \mu \mathrm{g})$, gentamicin $(30 \mu \mathrm{g})$, imipenem $(10 \mu \mathrm{g})$, levofloxacin $(5 \mu \mathrm{g})$, meropenem $(10 \mu \mathrm{g})$, nalidixic acid $(30 \mu \mathrm{g})$, netilmicin $(30 \mu \mathrm{g})$ and nitrofurantoin $(300 \mu \mathrm{g})$. Isolates were labeled as 'sensitive' and 'resistant' according to the guidelines of Clinical Laboratory Standard Institute (CLSI). Escherichia coli ATCC 25922 and Staphylococcus aureus ATCC 25923 were used as control strains for AST. ${ }^{13}$ Resistance to at least one agent in three or more antimicrobial categories was defined as multi drug resistance. ${ }^{14}$ 
Data collection and statistical analysis: A structured questionnaire was used for patient's demographic and clinical data. Descriptive statistical methods in SPSS (version 21.0 for Windows, SPSS $®$ Inc., Chicago, IL) were applied for data analysis. The antimicrobial resistance prevalence was calculated as the proportion of positive results against total sample. Nominal variables were shown as number of cases $(n)$ and percentage (\%). Test of significance was performed using the Chi-square $\left(X^{2}\right)$ test and variables including demographic and clinical characteristics of UTI patients were compared using cross-tabulation statistical methods. $P<0.05$ was considered statistically significant.

\section{RESULTS}

Culture yielded $537(42.8 \%)$ as positive out of 1255 clinically suspected UTI patients. Escherichia coli was the most common (61.6\%) gram negative isolate followed by Klebsiella spp. (22.5\%), Pseudomonas spp. (7.8\%) and Enterobacter spp. (2.6\%), while Staphylococcus aureus $(5.4 \%)$ was the only
Table-I: Frequency of culture-positive $(\mathrm{n}=537)$ and MDR $(n=420)$ uropathogens.

\begin{tabular}{lcc}
\hline Uropathogens & $\begin{array}{c}\text { Frequency of } \\
\text { culture-positive } \\
\text { isolates } n(\%)\end{array}$ & $\begin{array}{c}\text { Frequency of } \\
\text { MDR } \\
\text { isolates } n(\%)\end{array}$ \\
\hline Escherichia coli & $331(61.6)$ & $257(77.6)$ \\
Klebsiella spp. & $121(22.5)$ & $87(71.9)$ \\
Pseudomonas spp. & $42(7.8)$ & $38(90.5)$ \\
Staphylococcus aureus & $29(5.4)$ & $25(86.2)$ \\
Enterobacter spp. & $14(2.6)$ & $13(92.9)$ \\
\hline Total & $537(100)$ & $420(78.2)$ \\
\hline
\end{tabular}

gram positive isolate. Of 537 isolates, $420(78.2 \%)$ were found to be MDR with frequency distribution of Escherichia coli, Klebsiella spp., Pseudomonas spp., Staphylococcus aureus and Enterobacter spp. was $77.6 \%, 71.9 \%, 90.5 \%, 86.2 \%$ and $92.9 \%$ respectively (Table-I).

There was female preponderance (M:F; 1:1.97) among culture-positive cases and statistically highly significant $(\mathrm{P}=0.007)$. Age distribution revealed $252(20 \%)$ patients from paediatric age

Table-II: Correlation of UTI with demographic and clinical characteristics of the patients.

\begin{tabular}{|c|c|c|c|c|}
\hline Characteristics & lture-positive $n(\%)$ & Culture-negative $n(\%)$ & Total n(\%) & P-value \\
\hline \multicolumn{5}{|l|}{ Gender } \\
\hline Female & $356(66.3)$ & $422(58.8)$ & $778(62.0)$ & \multirow[t]{2}{*}{0.007} \\
\hline Male & $181(33.7)$ & $296(41.2)$ & $477(38.0)$ & \\
\hline \multicolumn{5}{|l|}{ Age } \\
\hline$<5$ years & $70(13.0)$ & $92(12.8)$ & $162(12.9)$ & \multirow[t]{7}{*}{0.994} \\
\hline $5-18$ years & $40(7.4)$ & $50(7.0)$ & $90(7.2)$ & \\
\hline 19 - 30 years & 95 (17.7) & $135(18.8)$ & $230(18.3)$ & \\
\hline $31-40$ years & 65 (12.1) & $92(12.8)$ & $157(12.5)$ & \\
\hline $41-50$ years & $71(13.2)$ & 98 (13.6) & 169 (13.5) & \\
\hline $51-60$ years & 83 (15.5) & $104(14.5)$ & $187(14.9)$ & \\
\hline$>60$ years & $113(21.0)$ & $147(20.5)$ & $260(20.7)$ & \\
\hline \multicolumn{5}{|l|}{ Chronic Medical Conditions } \\
\hline Hypertension & $108(20.1)$ & 140 (19.5) & $248(19.8)$ & 0.487 \\
\hline Diabetes mellitus & $139(25.8)$ & $92(12.8)$ & $231(18.4)$ & 0.004 \\
\hline Ischaemic heart disease & $20(3.7)$ & $32(4.4)$ & $52(4.1)$ & 0.364 \\
\hline Chronic obstructive pulmonary disease & $21(3.9)$ & $28(3.8)$ & $49(3.9)$ & 0.148 \\
\hline Chronic renal failure & $23(4.2)$ & $16(2.2)$ & $39(3.1)$ & 0.029 \\
\hline Asthma & $11(2.1)$ & $17(2.3)$ & $28(2.2)$ & 0.774 \\
\hline \multicolumn{5}{|l|}{ Clinical presentation } \\
\hline Dysuria & $432(80.5)$ & $559(77.8)$ & $991(79.0)$ & 0.372 \\
\hline Frequency & $296(55.1)$ & $375(52.2)$ & $671(53.5)$ & 0.143 \\
\hline Fever & $257(47.8)$ & $166(23.2)$ & $423(33.7)$ & 0.012 \\
\hline Urgency & $192(35.7)$ & $206(28.7)$ & 398 (31.7) & 0.271 \\
\hline Supra-pubic pain & $158(29.4)$ & $174(24.2)$ & $332(26.5)$ & 0.048 \\
\hline Nocturia & $84(15.6)$ & $98(13.6)$ & $182(14.5)$ & 0.153 \\
\hline Haematuria & $30(5.6)$ & $32(4.4)$ & $62(4.9)$ & 0.076 \\
\hline
\end{tabular}


group (up to 18 yrs.) and $1003(80 \%)$ adults with no significant difference in frequency of culturepositive cases ( $43.7 \%$ vs. $42.6 \%)$. Also there was no statistical significance in rate of isolation among different age groups. Co-morbid conditions like hypertension $(19.8 \%)$, diabetes mellitus $(18.4 \%)$, ischaemic heart disease $(4.1 \%)$, chronic obstructive pulmonary disease $(3.9 \%)$, chronic renal failure $(3.1 \%)$ and asthma $(2.2 \%)$ were noted among UTI patients with significant $(\mathrm{P}<0.05)$ association observed for diabetic and chronic renal failure patients. Among symptoms, dysuria (79\%), frequency $(53.5 \%)$, fever $(33.7 \%)$, urgency $(31.7 \%)$, supra-pubic pain (26.5\%), nocturia (14.5\%) and haematuria $(4.9 \%)$ were noted with fever and supra-pubic pain had significant $(P<0.05)$ association with UTI (Table-II).

MDR ranged from 3.7 to $88.1 \%$ and isolates were found to be moderate to highly resistant (28.6 to $92.9 \%)$ to commonly used antibiotics like ciprofloxacin, cotrimoxazole, azithromycin, nalidixic acid, cephalosporin and aztreonam (Table-III). Nitrofurantoin, amikacin, netilmicin, imipenem and meropenem were found to be better choice (resistance ranged from 2.4 to $32.2 \%$ ) for all isolates, except Pseudomonas spp. and Enterobacter spp. Nitrofurantoin resistance was $66.7 \%$ for Pseudomonas spp. while Enterobacter spp. showed moderate resistance to both imipenem (28.6\%) and meropenem $(42.9 \%)$.

\section{DISCUSSION}

Resistance to commonly used antibiotics is an emerging concern worldwide causing treatment failure in different infections including UTI. ${ }^{15}$ The present study highlights a baseline evidence on current situation of very high frequency of MDR uropathogens among hospitalized patients. It is no wonder that we found female preponderance which is an established fact that due to many inherent host factors females are more vulnerable to UTI. ${ }^{4}$ Diabetes and chronic renal failure were found to have statistically significant association with UTI and these findings corroborate well with other reports. ${ }^{16,17}$ Predisposition to UTI in diabetes results from several factors including increased susceptibility to uncontrolled glycaemia and defectivehostimmunity. Whilechronic renal failure is a risk factor for UTI due to metabolic disorders resulting in secondary immunodeficiency.

Fever and supra pubic pain had significant association among others as presentations of UTI. Fever usually denotes pyelonephritis and more commonly observed in paediatric patients while supra pubic pain indicates cystitis which is common among lower UTI. ${ }^{18}$ Escherichia coli was the most frequent isolate followed by Klebsiella spp., which is consistent with many previous studies. ${ }^{4,6,8,10}$ In fact, E. coli stands alone for around $70 \%$ as the etiological agent of both community and hospital acquired UTI. ${ }^{4}$ We found

Table-III: Frequency of antimicrobial resistance of uropathogens $(n=537)$.

\begin{tabular}{lcccccc}
\hline $\begin{array}{c}\text { Antimicrobial } \\
\text { Agents }\end{array}$ & $\begin{array}{c}\text { Escherichia coli } \\
(n=331)\end{array}$ & $\begin{array}{c}\text { Klebsiella spp. } \\
(n=121)\end{array}$ & $\begin{array}{c}\text { Pseudomonas spp. } \\
(n=42)\end{array}$ & $\begin{array}{c}\text { Staphylococcus } \\
\text { aureus }(n=29)\end{array}$ & $\begin{array}{c}\text { Enterobacter } \\
\text { spp. }(n=14)\end{array}$ & $\begin{array}{c}\text { Total } \\
\text { MDR } n(\%)\end{array}$ \\
\hline Cefixime & $187(56.5)$ & $74(61.2)$ & $25(59.5)$ & $12(41.4)$ & $11(78.6)$ & $309(57.5)$ \\
Ceftriaxone & $166(50.2)$ & $69(57.0)$ & $16(38.1)$ & $3(10.3)$ & $7(50.0)$ & $261(48.6)$ \\
Ceftazidime & $128(38.7)$ & $53(43.8)$ & $12(28.6)$ & $9(31.0)$ & $10(71.4)$ & $212(39.5)$ \\
Cefepime & $158(47.7)$ & $57(47.1)$ & $13(31.0)$ & $10(34.5)$ & $10(71.4)$ & $248(46.2)$ \\
Amikacin & $29(8.8)$ & $16(13.2)$ & $2(4.8)$ & $2(6.9)$ & $3(21.4)$ & $52(9.7)$ \\
Gentamicin & $134(40.5)$ & $55(45.5)$ & $22(52.4)$ & $13(44.8)$ & $9(64.3)$ & $233(43.4)$ \\
Imipenem & $9(2.7)$ & $5(4.1)$ & $1(2.4)$ & $1(3.4)$ & $4(28.6)$ & $20(3.7)$ \\
Meropenem & $8(2.4)$ & $9(7.4)$ & $2(4.8)$ & $2(6.9)$ & $6(42.9)$ & $27(5.0)$ \\
Ciprofloxacin & $189(57.1)$ & $46(38.0)$ & $14(33.3)$ & $18(62.1)$ & $10(71.4)$ & $277(51.6)$ \\
Levofloxacin & $173(52.3)$ & $41(33.9)$ & $12(28.6)$ & $4(13.8)$ & $10(71.4)$ & $240(44.7)$ \\
Azithromycin & $178(53.8)$ & $66(54.5)$ & $20(47.6)$ & $18(62.1)$ & $9(64.3)$ & $291(54.2)$ \\
Cefuroxime & $154(46.5)$ & $70(57.9)$ & $26(61.9)$ & $18(62.1)$ & $9(64.3)$ & $277(51.6)$ \\
Aztreonam & $131(39.6)$ & $61(50.4)$ & $12(28.6)$ & $24(82.8)$ & $13(92.9)$ & $241(44.9)$ \\
Cotrimoxazole & $192(58.0)$ & $66(54.5)$ & $35(83.3)$ & $20(69.0)$ & $8(57.1)$ & $321(59.8)$ \\
Nitrofurantoin & $68(20.5)$ & $39(32.2)$ & $28(66.7)$ & $5(17.2)$ & $3(21.4)$ & $143(26.6)$ \\
Netilmicin & $60(18.1)$ & $38(31.4)$ & $10(23.8)$ & $8(27.6)$ & $3(21.4)$ & $119(22.2)$ \\
Nalidixic Acid & $292(88.2)$ & $110(90.9)$ & $35(83.3)$ & $24(82.8)$ & $12(85.7)$ & $473(88.1)$ \\
\hline N.B. Figures & & & & &
\end{tabular}

N.B. Figures in the parentheses indicate percentage. 
Staph aureus as only gram positive uropathogen and it coincides with other report. ${ }^{19}$ Although coagulase negative Staph saprophyticus has been reported as frequent uropathogen usually from patients attending outpatient or antenatal clinics but it contradicts with ours because we included hospital admitted cases where Staph aureus is a common pathogen. ${ }^{4,5,17}$

High prevalence of MDR uropathogens with moderate to high resistance to most of the common antimicrobial classes including cephalosporin, quinolone and fluoroquinolones, macrolide, cotrimoxazole and injectable drugs like aztreonam and gentamicin was observed in the present study which is higher in comparison to our previous report. ${ }^{5}$ This difference can be correlated with inclusion of hospital acquired UTI in the present setting where there is high frequency of MDR uropathogens. ${ }^{20}$ Comparable prevalence rate of MDR uropathogens to commonly prescribed antimicrobials has also been reported by some recent studies. ${ }^{21,22}$

We found nitrofurantoin and amikacin as better choice except Pseudomonas spp. which is consistent with our previous report and other recent studies. ${ }^{5,23,24}$ Reasons for the emergence of low resistance to nitrofurantoin are not fully understood, but likely include its restricting use, limited systemic absorption and the need for multiple genetic mutations in bacteria to develop resistance. The most important and alarming findings of the present study are variable degree of resistance to carbapenems ranging from 2.4 to $42.9 \%$ shown by different isolates which corroborates with others. ${ }^{23,25}$ Carbapenems are the latest developed $\beta$-lactam containing broad spectrum antibiotics usually reserved for MDR pathogens. Lately, the dissemination of community acquired $E$. coli capable of producing ESBLs that can hydrolyze almost all $\beta$-lactams except carbapenems has been reported worldwide; consequently, the use and abuse of carbapenems have increased greatly with emergence of carbapenem resistance as a serious concern. Evidence based choice of antibiotics from in-vitro antimicrobial susceptibility test may reduce the risk of this alarming situation when there is current shortage of effective therapies, lack of successful preventive measures and of course very slow development of novel treatment options. Further, practice of Antibiotic Stewardship Programs (ASPs) in hospitals can help minimizing the problem of antibiotic resistance and it has been recommended by the Centers for Disease Control and Prevention (CDC). ASPs refer to optimizing the use of antibiotics to effectively treat infections, protect patients from harms caused by unnecessary antibiotic use and combat antibiotic resistance. The programs have at least seven core components to help clinicians to improve clinical outcomes by improving antibiotic prescribing practice (https://www.cdc.gov/antibiotic-use/ healthcare/pdfs/hospital-core-elements-H.pdf).

Limitations of the study: First of all, it was a single center study, so the findings cannot be generalized in the national context. Second, the detailed clinical classification of UTI patients could not be mentioned. Third, the genotypic or phenotypic categorization of MDR uropathogens were not established.

\section{CONCLUSIONS}

Increasing frequency of MDR uropathogens is a global concern and has been reinforced by our findings. Commonly prescribed antibiotics including third generation cephalosporin and fluoroquinolones have shown to be poorly efficacious while nitrofurantoin, netilmicin and amikacin still hold some promise. Although the reserve drugs like carbapenems are still the best choice in treating the hospital acquired UTI but emergence of carbapenem resistance is a great concern. Antibiotic Stewardship Programs (ASPs) can only optimize the use of antibiotics to effectively treat infections and to improve clinical outcomes. We strongly advocate the introduction of ASPs in the tertiary care hospitals in Bangladesh to combat antibiotic resistance.

Acknowledgements: The authors would like to express their sincere gratitude to study participants.

\section{Conflict of interest: None.}

Grant Support \& Financial Disclosures: None.

\section{List of Abbreviations:}

UTI: Urinary tract infection, MDR: Multi drug resistant,

CLSI: Clinical and Laboratory Standards Institute, CFU: Colony forming unit,

HPF: High power field, AST: Antimicrobial susceptibility testing,

ASPs: Antibiotic Stewardship Programs,

SZMCH: Shaheed Ziaur Rahman Medical College Hospital. 


\section{REFERENCES}

1. Ozturk R, Murt A. Epidemiology of urological infections: A global burden. World J Urol. 2020. doi: 10.1007/s00345-01903071-4

2. Geerlings SE. Clinical Presentations and Epidemiology of Urinary Tract Infections. Microbiol Spectr. 2016;4(5). doi: 10.1128/microbiolspec.UTI-0002-2012

3. McLellan LK, Hunstad DA. Urinary Tract Infection: Pathogenesis and Outlook. Trends Mol Med. 2016;22(11):946957. doi: 10.1016/j.molmed.2016.09.003

4. Flores-Mireles A, Walker J, Caparon M, Hultgren SJ. Urinary tract infections: epidemiology, mechanisms of infection and treatment options. Nat Rev Microbiol. 2015;13:269-284. doi: $10.1038 /$ nrmicro3432

5. Haque R, Akter ML, Salam MA. Prevalence and susceptibility of uropathogens: A recent report from a teaching hospital in Bangladesh. BMC Res Notes. 2015;8(1). doi: 10.1186/s13104015-1408-1

6. Shiralizadeh S, Taghizadeh S, Asgharzadeh M, Shokouhi B, Gholizadeh P, Rahbar M, et al. Urinary tract infections: raising problem in developing countries. Rev Med Microbiol. 2018;29(4):159-165. doi: 10.1097/MRM.0000000000000144

7. Ahmed I, Rabbi MB, Sultana S. Antibiotic resistance in Bangladesh: A systematic review. Int J Infect Dis. 2019;80:54-61. doi: 10.1016/j.ijid.2018.12.017

8. Al-Zahrani J, Al Dossari K, Gabr AH, Ahmed A, Al Shahrani SA, Al-GhamdiS. Antimicrobial resistance patterns of Uropathogens isolated from adult women with acute uncomplicated cystitis. BMC Microbiol. 2019;19:237. doi: 10.1186/s12866-019-1612-6

9. Fuller CE, Threatte GA, Henry JB. Basic examination of the urine. In Clinical Diagnosis and Management by Laboratory Methods, 20th ed.; Henry JB, Davey FR, Herman CJ, McPherson RA, Pincus MR, Threatte GA, Woods GL, Eds.; WB Saunders: Philadelphia, PA, USA, 2001;p.367-402.

10. Akter ML, Haque R, Salam MA. Comparative evaluation of chromogenic agar medium and conventional culture system for isolation and presumptive identification of uropathogens. Pak J Med Sci 2014;30(5):1033-1038. doi: 10.12669/pjms.305.5243

11. Collee JG, Miles RS, Watt B. In: Mackie \& McCartney's Practical Medical Microbiology, Collee JG, Fraser AG, Marmion BP, Simmons A, Eds.; Churchil Livingstone, New York, 1996; p131-149.

12. Bauer AW, Kirby WM, Sherris JC, Turck M. Antibiotic susceptibility testing by a standardized single disk method Tech Bull Regist Med Technol. 1966;36(3):49-52.

13. CLSI. Performance Standards for Antimicrobial Susceptibility Testing. 28th ed. CLSI supplement M100. Wayne, PA: Clinical and Laboratory Standards Institute; 2018. www.clsi.org

14. Magiorakos AP, Srinivasan A, Carey RB, Carmeli Y, Falagas $\mathrm{ME}$, Giske CG, et al. Multidrug-resistant, extensively drugresistant and pandrug-resistant bacteria: An international expert proposal for interim standard definitions for acquired resistance. Clin Microbiol Infect. 2012;18(3):268-281. doi: 10.1111/j.1469-0691.2011.03570.x

15. Huttner A, Harbarth S, Carlet J, Cosgrove S, Goossens $\mathrm{H}$, Holmes A, et al. Antimicrobial resistance: a global view from the 2013 World Healthcare-Associated Infections Forum. Antimicrob Resist Infect Control. 2013;2:31. doi 10.1186/2047-2994-2-31

16. de Lastours V, Foxman B. Urinary tract infection in diabetes: epidemiologic considerations. Curr Infect Dis Rep. 2014;16(1):389. doi: 10.1007/s11908-013-0389-2

17. Sobotova D. Urinary tract infections and chronic renal failure. Vnitr Lek. 2011;57(7-8):626-630.

18. Lee SJ. Clinical Guideline for Childhood Urinary Tract Infection (Second Revision). Child Kidney Dis. 2015;19(2):56-64. doi: 10.3339/chikd.2015.19.2.56
19. Aliero AA, Tibyangye J, Maniga JN, Wampande E, Kato CD, Agwu E, et al. Prevalence of Bacterial Urinary Tract Infections and Associated Factors among Patients Attending Hospitals in Bushenyi District, Uganda. Int J Microbiol. 2019;4246780. doi: $10.1155 / 2019 / 4246780$

20. Bardoloi V, Yogeesha Babu KV. Comparative study of isolates from community-acquired and catheter-associated urinary tract infections with reference to biofilm-producing property, antibiotic sensitivity and multi-drug resistance. J Med Microbiol. 2017;66(7):927-936. doi: 10.1099/jmm.0.000525

21. Nahar A, Hasnat S, Akhter H, Begum N. Evaluation of antimicrobial resistance pattern of uropathogens in a tertiary care hospital in Dhaka city, Bangladesh. South East Asia J Public Health. 2017;7(2):12-18. doi: 10.3329/seajph.v7i2.38851

22. Vazouras K, Velali K, Tassiou I, Anastasiou-Katsiardani A, Athanasopoulou K, Barbouni A, et al. Treatment and antimicrobial Resistance in Children with Urinary Tract Infections. J Glob Antimicrob Resist. 2019;20:4-10. doi: 10.1016/j. jgar.2019.06.016

23. Pirkani GS, Awan MA, Abbas F, Din M. Culture and PCR based detection of bacteria causing urinary tract infection in urine specimen. Pak J Med Sci. 2020;36(3):391-395. doi: 10.12669/ pjms.36.3.1577

24. Demir M, Kazanasmaz HJ. Uropathogens and antibiotic resistance in the community and hospital-induced urinary tract infected children. J Glob Antimicrob Resist. 2020;20:68-73. doi: 10.1016/j.jgar.2019.07.019

25. Ann AE, Khaled MA, Mahmoud AY, Nadia AH. Prevalence of carbapenem resistance among multidrug-resistant Gramnegative uropathogens. Arch Pharm Sci ASU. 2018;2(2):70-77. doi: $10.21608 /$ aps.2018.18736

\section{Author's Contributions}

CD \& MAS: conceived, designed and editing of the manuscript.

MAR \& CD: data collection, analysis and manuscript writing.

MAS: responsible for the accuracy and integrity of the work.

All authors have read and approved the final form of the manuscript.

Authors:

1. Chandrika Dasgupta, MBBS.

Intern Doctor,

Shaheed Ziaur Rahman Medical College Hospital,

Bogura, Bangladesh.

2. Md. Abdur Rafi, MBBS.

Intern Doctor,

Rajshahi Medical College Hospital, Rajshahi, Bangladesh.

3. Md. Abdus Salam, PhD, FRCP (UK).

Department of Microbiology,

Present Address: Department of Basic Medical Sciences,

Kulliyyah (Faculty) of Medicine,

International Islamic University Malaysia,

25200 Kuantan, Pahang, Malaysia.

1,3: Shaheed Ziaur Rahman Medical College,

Bogura, Bangladesh.

Correspondence:

Dr. Md. Abdus Salam

Professor of Parasitology, Department of Basic Medical Sciences,

Kulliyyah (Faculty) of Medicine,

International Islamic University Malaysia.

25200 Kuantan, Pahang, Malaysia.

E-mail: abdussalam@iium.edu.my 